\title{
Crescimento de cultivares de cenoura em diferentes ambientes
}

\author{
Gustavo Antônio Mendes Pereira', Maxwel Coura Oliveira², \\ Renan Rodrigues Braga*1, Daniel Valadão Silva', Altino Júnior Mendes Oliveira ${ }^{3}$, \\ José Sebastião Cunha Fernandes ${ }^{3}$, Valter Carvalho de Andrade Júnior ${ }^{3}$ \\ 'Universidade Federal de Viçosa, Viçosa, MG, Brasil \\ 2University of Nebraska-Lincoln, Estados Unidos \\ 3Universidade Federal dos Vales do Jequitinhonha e Mucuri, Teófilo Otoni, MG, Brasil \\ *Autor correspondente, e-mail: granderenan@gmail.com
}

\section{Resumo}

A cenoura (Daucus carota) é a quinta hortaliça mais cultivada no Brasil, apresentando produtividade média de apenas 32,1 † ha-1 com potencial produtivo para alcançar $80 \mathrm{~h} \mathrm{ha}^{-1}$. Uma das causas apontadas para esta baixa produtividade é o plantio de cultivares não adaptadas aos ambientes encontrados no Brasil. Sabendo da importância dos efeitos da interação genótipo e ambiente, objetivou-se avaliar o crescimento de cultivares de cenoura no cultivo de outono-inverno em dois municípios com características edafo-climáticas distintas do Alto Vale do Jequitinhonha, MG. Foram cultivadas seis cultivares de cenoura (Brasília, Nantes, Kuronan, Esplanada, Planalto e Tornado) em dois ambientes (Couto de Magalhães de Minas e Diamantina). As plantas foram amostradas semanalmente e foram ajustadas equações de regressão a partir do tempo. Para a altura de plantas ajustou-se modelos lineares para Couto de Magalhães de Minas e para Diamantina modelos quadráticos, já no comprimento de raízes o comportamento se inverteu. Essa diferença mostra que ambientes distintos alteram o padrão de crescimento. Os ambientes distintos resultaram em diferentes comportamentos dos cultivares de cenoura, sendo que em Couto de Magalhães de Minas a produtividade foi maior. Independente do ambiente de cultivo a variedade Nantes apresentou menor produtividade. O cultivar Planalto é mais indicada para plantio em Diamantina e a Kuronan e Planalto para Couto Magalhães de Minas.

Palavras-chave: análise de crescimento, Daucus carota, produtividade

\section{Growth of carrot cultivars in different environments}

\begin{abstract}
The carrot (Daucus carota) is the fifth most widely grown vegetable crop in Brazil, with an average yield of only 32.1 t ha-1 with productive potential to reach 80 tha-1. One reason cited for this low productivity is planting varieties not adapted to the environments found in Brazil. Knowing the importance of genotype and environment interaction, aimed to evaluate the growth of carrot cultivars in autumn-winter crop in two counties with different soil and climatic characteristics of the Upper Valley Jequitinhonha, MG. Six carrot cultivars (Brasilia, Nantes, Kuronan, Esplanade, Plateau and Tornado) were grown in two environments (Couto de Magalhães of Mines and Diamantina). Plants were sampled weekly and regression equations were adjusted from time. For plant height set to linear Couto de Magalhães de Diamantina Minas and quadratic models, as in root length models the behavior was reversed. This difference shows that different environments alter the pattern of growth. The distinct environments resulted in different behaviors of cultivars of carrot, and in Couto de Magalhães de Minas productivity was higher. Regardless of the growing environment to Nantes variety showed lower productivity. The Plateau cultivar is best suited for planting in the Diamantina and Kuronan and Plateau to Couto Magalhães de Minas.
\end{abstract}

Keywords: Daucus carota, growth analysis, yield 


\section{Introdução}

A cenoura (Daucus carota) é a quinta hortaliça mais cultivada no Brasil, sendo encontrada em larga escala nas regiões Sul, Sudeste e Nordeste do país. A produtividade média nacional é baixa, 32,1 † ha-1 (EMBRAPA, 2008), embora algumas regiões alcancem valores superiores a $80 \mathrm{t} \mathrm{ha}^{-1}$ (EMBRAPA, 2007). Um dos fatores que contribuem para a reduzida produtividade é o plantio de cultivares não adaptadas aos vários ambientes encontrados no Brasil (Lopes et al., 2008), sendo que a simples escolha adequada do cultivar para cada região, pode representar enormes ganhos econômicos para o agricultor.

A recomendação de genótipos é realizada para extensas faixas de ambientes, com base na sua produtividade média. Assim não se considera a adaptação específica de cada genótipo em cada local (Cruz \& Castoldi, 1991). Estudos da interação genótipo x ambiente têm sido realizados em diversas hortaliças, tais como: alface (Figueiredo, 2004), tomate (Gualberto, 2002) e batata (Peixoto et al., 2002). Entretanto, na cultura da cenoura, não se tem observado pesquisas com relação à interação genótipo x ambiente (Oliveira et al., 2005).

Existem diferenças microclimáticas dentro de uma mesma zona agrícola onde se espera condições ecológicas aparentemente semelhantes (Possas et al., 2012). Os efeitos destas diferenças microclimáticas sobre o desempenho de determinada cultura são de grande importância, assim sendo, antes de se fazer recomendações definitivas de genótipos em locais distintos, é necessário a verificação do desempenho dos cultivares disponíveis para tal região.

A análise de crescimento possibilita identificar características das plantas associadas a adaptações e condições de estresse, bem como fornece informações sobre os efeitos dos ambientes sobre o desenvolvimento e produção de espécies e, ou cultivares (Andrade et al., 2005). Usualmente, a medida sequencial do acúmulo de matéria orgânica, considerando o peso das partes secas da planta (frutos, caule, folhas e outros) é o fundamento da análise de crescimento (Fontes et al., 2005).
A hipótese desta pesquisa é que - padrão de crescimento da cenoura é modificado quando a hortaliça é cultivada em diferentes ambientes. Neste sentido, objetivou-se avaliar o crescimento de cultivares de cenoura no cultivo de outono-inverno em dois municípios com características edafo-climáticas distintas do Alto Vale do Jequitinhonha, MG.

\section{Material e Métodos}

Realizaram-se dois experimentos em locais distintos, durante os meses de maio a novembro de 2011. O primeiro no Campus Experimental Rio Manso da Universidade Federal dos Vales do Jequitinhonha e Mucuri - UFVJM, localizada no município de Couto de Magalhães

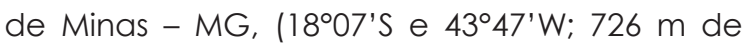
altitude), índice pluviométrico médio anual de $1.269 \mathrm{~mm}$, temperatura média anual de 19,4 ${ }^{\circ} \mathrm{C}$ e solo classificado como Latossolo Vermelho Distrófico. O segundo experimento foi conduzido no Setor de Olericultura da UFV JM - Campus JK, localizado no município de Diamantina, MG, (1812'S e 4334'W; $1387 \mathrm{~m}$ de altitude) com temperatura média anual de $18,0^{\circ} \mathrm{C}$, índice médio pluviométrico anual de 1404,7 mm, localizado sobre um Neossolo Quartzarênico Órtico Típico. Na Tabela 1 encontram-se as temperaturas mínimas, máximas, médias, e precipitação pluviométrica, observadas no período para os dois municípios.

O delineamento experimental adotado foi de blocos casualizados com três repetições, em esquema de parcela subdividida no tempo, sendo cada sub-parcela constituída por cinco plantas. As parcelas foram constituídas por nove épocas de coletas de plantas (coletas semanais a partir do momento do desbaste, até o dia da colheita), e as subparcelas por seis genótipos de cenoura (Brasília, Nantes, Kuronan, Esplanada, Planalto e Tornado).

As áreas experimentais foram submetidas à aração e gradagem, em seguida foram feitos canteiros, sendo as parcelas constituídas de uma área de $3,75 \mathrm{~m}^{2}$ e área útil de $2 \mathrm{~m}^{2}$, com 4 fileiras de plantio, espaçadas por 0,24 m.

A correção da acidez e a fertilização do solo foram feitas de acordo com os resultados das análises de solo das áreas experimentais (Tabela 
Tabela 1. Temperaturas máximas, mínimas, médias $\left({ }^{\circ} \mathrm{C}\right)$ e precipitação pluviométrica $(\mathrm{mm})$ referente aos meses de maio a novembro de 2011

\begin{tabular}{ccccccccc}
\hline Variável & Local & Mai & Jun & Jul & Ago & Set & Out & Nov \\
\hline Temperatura & Diamantina & 21,5 & 22,2 & 22,3 & 25,4 & 25,2 & 23,1 & 22,8 \\
Máxima & Couto de M. & 25,2 & 26,4 & 26,6 & 29,5 & 29,1 & 27,1 & 26,2 \\
\hline Temperatura & Diamantina & 16,5 & 17,1 & 16,9 & 19,2 & 18,8 & 18,8 & 18,6 \\
Média & Couto de M. & 18,6 & 19,2 & 18,7 & 20,4 & 20,3 & 22,0 & 21,7 \\
\hline Temperatura & Diamantina & 11,4 & 12,1 & 11,4 & 13,0 & 12,5 & 14,5 & 14,3 \\
Mínima & Couto de M. & 12,0 & 12,1 & 10,9 & 11,3 & 11,5 & 16,9 & 17,2 \\
\hline \multirow{2}{*}{ Precipitação } & Diamantina & 0,4 & 0,3 & 0,01 & 0,5 & 0,1 & 178,8 & 342,5 \\
& Couto de M. & 0,3 & 0,01 & 5,7 & 0,01 & 0,01 & 82,6 & 269,4 \\
\hline Fonte: INMEt, 2011 & & & & & & & &
\end{tabular}

2), seguindo critérios da Comissão de Fertilidade do Solo do Estado de Minas Gerais (CFSEMG, 1999). Em Diamantina realizou-se a calagem com $1.030 \mathrm{~kg} \mathrm{ha}^{-1}$ de calcário dolomítico e na adubação de plantio foram adicionados $240 \mathrm{~kg}$ $\mathrm{ha}^{-1}$ de $\mathrm{P}_{2} \mathrm{O}_{5^{\prime}} 128 \mathrm{~kg} \mathrm{ha}^{-1}$ de $\mathrm{K}_{2} \mathrm{O}$ e $36 \mathrm{~kg} \mathrm{ha}^{-1} \mathrm{de}$ $\mathrm{N}$, nas fontes, supersimples, cloreto de potássio e sulfato de amônia, respectivamente. E em cobertura foram realizadas duas aplicações: no momento do desbate e aproximadamente
60 dias após semeadura foram fornecidos 96 $\mathrm{kg} \mathrm{ha}^{-1}$ de $\mathrm{K}_{2} \mathrm{O}$ e $42 \mathrm{~kg} \cdot \mathrm{ha}^{-1}$ de N. Em Couto de Magalhães de Minas não foi necessário adição de calcário, sendo a adubação de plantio constituída de $320 \mathrm{~kg} \mathrm{ha}^{-1}$ de $\mathrm{P}_{2} \mathrm{O}_{5}, 64 \mathrm{~kg} \mathrm{ha}^{-1}$ de $\mathrm{K}_{2} \mathrm{O}$ e $36 \mathrm{~kg} \mathrm{ha}^{-1}$ de $\mathrm{N}$, utilizando as mesmas fontes de Diamantina. E em cobertura nas mesmas datas foram adicionados $48 \mathrm{~kg} \mathrm{ha}^{-1}$ de $\mathrm{K}_{2} \mathrm{O}$ e 42 $\mathrm{kg} \mathrm{ha}^{-1}$ de N.

Tabela 2. Resultados da análise química e física de amostras de solo das áreas experimentais. UFV JM, 2014.

\begin{tabular}{|c|c|c|c|c|c|c|c|c|c|c|}
\hline Ambientes & $\mathrm{pH}$ & $P$ & K & $\mathrm{Ca}$ & $\mathrm{Mg}$ & $\mathrm{Al}$ & \multirow{2}{*}{\multicolumn{2}{|c|}{ cmolc. $\mathrm{dm}^{-3}$}} & $\mathrm{~T}$ & $t$ \\
\hline & 5 & g.dn & - & & & & & & & \\
\hline \multirow{2}{*}{ Couto de M. } & $\begin{array}{l}5,6 \\
5,0\end{array}$ & $\begin{array}{l}2,5 \\
6,1\end{array}$ & $\begin{array}{l}12,6 \\
111\end{array}$ & $\begin{array}{l}0,7 \\
2,4\end{array}$ & $\begin{array}{l}0,45 \\
1,20\end{array}$ & $\begin{array}{l}0,12 \\
0,02\end{array}$ & $\begin{array}{l}1,9 \\
2,4\end{array}$ & $\begin{array}{l}1,18 \\
3,89\end{array}$ & $\begin{array}{l}3,08 \\
6,29\end{array}$ & $\begin{array}{l}1,30 \\
3,91\end{array}$ \\
\hline & V & $\mathrm{Fe}$ & $\mathrm{Cu}$ & Zn & $\mathrm{Mn}$ & B & Areia & Silte & \multicolumn{2}{|r|}{ Argila } \\
\hline Diamantina & 38 & 202 & 0.59 & 4.41 & 189 & 209 & 86 & 3 & & 11 \\
\hline Couto de M. & 62 & 43,3 & 1,28 & 1,2 & 233 & 0,14 & 60 & 13 & & 27 \\
\hline
\end{tabular}

As semeaduras foram realizadas diretamente no local definitivo de forma manual, no dia 26 de agosto de 2011. O desbaste foi efetuado aos 35 dias após semeadura, mantendo-se $5 \mathrm{~cm}$ de distância entre plantas na fileira.

Aos 40 DAS realizou-se o controle das plantas daninhas aplicando 1,6 e 1,8 $\mathrm{L} \mathrm{ha}^{-1}$ do herbicida AFALON SC ${ }^{\circledR}$ em Diamantina e Couto de Magalhães de Minas, respectivamente, respeitando as características texturais de cada solo. Durante todo o período de condução não foi necessário o controle de pragas e doenças. Os experimentos foram irrigados conforme estimativa da evapotranspiração da cultura, sempre que necessário.

Cinco plantas por parcela foram amostradas semanalmente a partir do momento do desbaste, até o dia da colheita 198 dias após semeadura) avaliando-se as seguintes características: altura da parte aérea (ALT); comprimento de raiz (COMP) utilizando-se régua graduada em mm; matéria seca da raiz (MSR), matéria seca total (MST), por meio da lavagem do material vegetal em água destilada e secagem em estufa com circulação forçada de ar, a $65^{\circ} \mathrm{C}$, até peso constante. A determinação da matéria seca da raiz e total foi realizada em balança eletrônica com precisão de 0,0001 g. Determinou-se também o índice de colheita (IC - Rendimento econômico / Rendimento biológico x 100) e produtividade (PROD). Para o índice de colheita (IC) utilizou-se método proposto por Floss (2006), no qual o rendimento biológico $(\mathrm{RB})=$ matéria seca total $(\mathrm{kg}) \times$ número de plantas por ha e o rendimento econômico 
$(\mathrm{RE})=$ matéria seca de raiz $(\mathrm{kg}) \times$ número de plantas por ha. O rendimento ecológico indica a produção de matéria das plantas como um todo e o rendimento econômico apenas a produção do órgão de interesse econômico.

Os resultados obtidos foram submetidos à análise de variância, de forma que para os cultivares, foi escolhido um modelo de regressão que melhor explicou os efeitos das épocas de avaliação sobre as variáveis consideradas com base no comportamento biológico do fenômeno, na significância dos coeficientes $(p<0,05)$ e no coeficiente de determinação.

Os cultivares cujos modelos escolhidos são idênticos foram agrupadas e seus valores médios foram utilizados para ajustar a equação de regressão. Estas equações ajustadas foram comparadas pelo teste de identidade de modelo para verificar a hipótese de nulidade de que as equações são iguais estatisticamente e quando nenhuma diferença significativa foi encontrada entre os modelos, os mesmos foram agrupados e representados pela mesma linha de tendência (Regazzi, 1999).

\section{Resultados e Discussão}

Para a análise da altura de parte aérea de plantas (ALT), ajustaram-se equações de regressão com modelos lineares para Couto de Magalhães de Minas e para Diamantina modelos quadráticos (Tabela 3). Essa diferença mostra que ambientes distintos alteram o padrão de comportamento dos cultivares. Em Couto de Magalhães de Minas observou-se maiores valores no final do ciclo, já em Diamantina as plantas estabilizaram seu crescimento próximo aos 42, 49 e 56 dias após desbaste (DAD) (Figura 11 e 111, A e B). Tal comportamento pode ser explicado pelos maiores valores de temperatura, observados em Couto de Magalhães de Minas, sendo que taxas fotossintéticas mais altas são observadas em respostas às "temperaturas ótimas", que são as faixas em que as máximas capacidades fotossintéticas são atingidas.

De acordo com Machado et al. (2005), para a maioria das plantas $\mathrm{C}_{3}$, como a cenoura, esta temperatura ótima se encontra próxima aos $25^{\circ} \mathrm{C}$, valor mais próximo das temperaturas médias mensais encontradas em Couto de
Magalhães de Minas (Tabela 1). Embora, outros fatores não mensurados também possam justificar tal diferença, como a intensidade luminosa, textura do solo, umidade do ar e etc.

Para o comprimento radicular (COMP) da cenoura em Couto de Magalhães de Minas houve ajuste quadráticos e, em Diamantina $\circ$ ajuste foi linear (Tabela 3). As características físicas do solo, $27 \%$ de argila em Couto Magalhães de Minas em comparação aos $11 \%$ em Diamantina (Tabela 2), podem ter imposto resistência ao crescimento de raízes, justificando tal resultado. Diversos autores relatam que a compactação do solo resulta em menor desenvolvimento radicular (Collares et al., 2008; Reinert et al., 2008; Richart et al., 2005). Porém, mesmo com os menores comprimentos de raízes, todos os cultivares estão dentro da "classe 22", que enquadra as raízes consideradas de maior comprimento para comercialização (CEAGESP, 2000).

Para matéria seca de raiz (MSR) e total (MST) observou-se comportamentos semelhantes, evidenciando que grande parte dos fotoassimilados são direcionados para a produção de raízes (Figuras $1 \mathrm{~V}$ e $1 \mathrm{VI}$ e 2l E 2II), principalmente na fase final do ciclo da cultura.

Os cultivares não apresentaram diferenças em relação ao índice de colheita (IC) em nenhum dos ambientes pelo teste de identidade de modelo, tendo ajustado o modelo linear em ambos ambientes. Entre ambientes 0 IC foi semelhante em ambos os locais (Tabela 3). Estes resultados mostram que os variados ambientes e cultivares não interferem na habilidade da planta de cenoura em acumular reservas nas raízes, sendo que o índice de colheita expressa a eficiência de translocação dos produtos da fotossíntese para os órgãos de importância econômica (Bezerra et al., 2008).

Houve ajuste do modelo quadrático para a produtividade (PROD) (Tabela 3). Entre ambientes observou-se que todas os cultivares obtiveram maior produtividade em Couto Magalhães de Minas (Figura $2 \mathrm{~V}$ e $2 \mathrm{VI}$ ), observando substanciais diferenças, como para - cultivar Kuronan que teve sua produtividade aumentada em $46 \%$ em relação ao cultivo em Diamantina. Este comportamento distinto em cada ambiente, demonstra que mesmo em 
áreas próximas, consideradas climaticamente semelhantes, pode haver microclimas onde o cultivo da cenoura é mais indicado. Um dos fatores que pode ter contribuído para maior produtividade pode está associada as maiores temperaturas em Couto Magalhaes de Minas, fato também verificado por Resende et al. (2005) que relatam influência da temperatura na produtividade da cenoura.

Em Couto de Magalhães de Minas observou-se que a cv. Nantes apresentou menor altura da parte aérea, comportamento semelhante ocorreu em Diamantina com a cv. Tornado (Figura 11 e 11l). Para comprimento de raízes apenas em Couto Magalhães de Minas houve diferença entre os materiais, com superioridade para a cv. Esplanada.

Ao se avaliar a matéria seca de raízes e total, o ajuste do modelo quadrático foi utilizado em todas as regressões (Tabela 3), sendo que em Couto de Magalhães de Minas os cultivares que obtiveram valores mais elevados foram, (l)

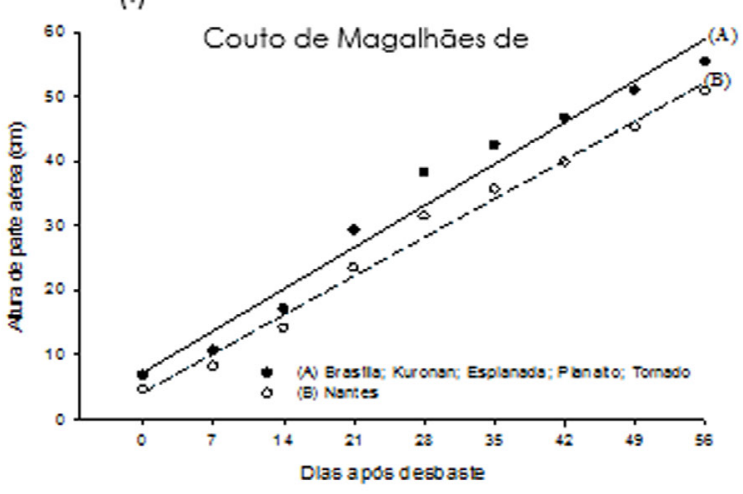

(III)

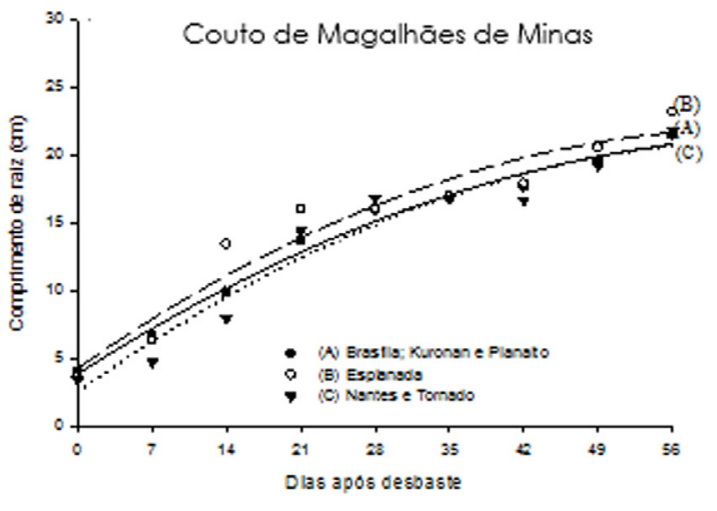

(V)

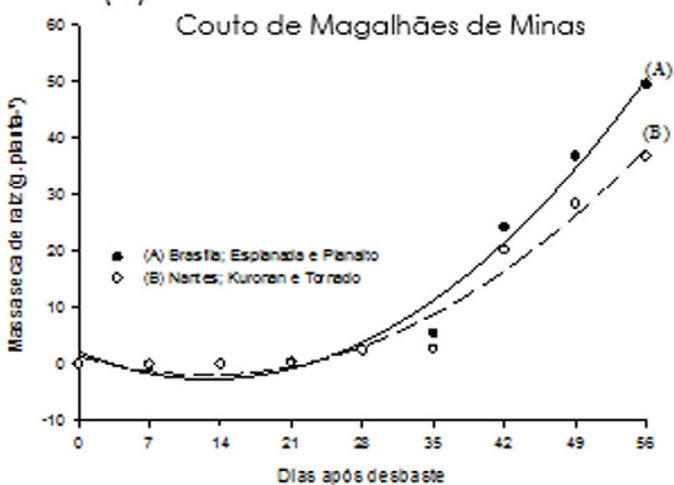

(II)

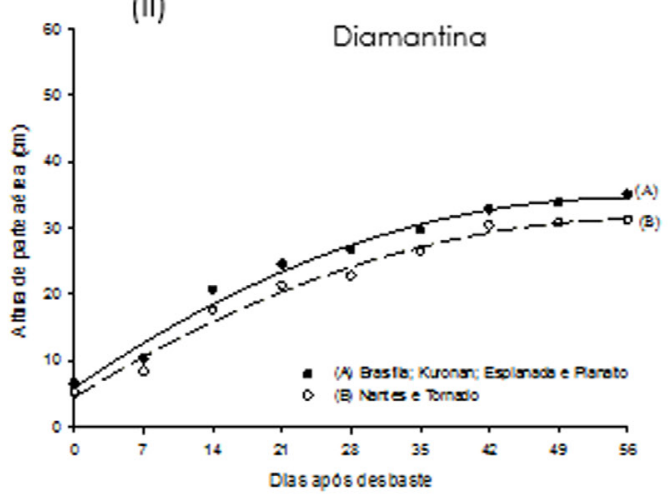

(IV)

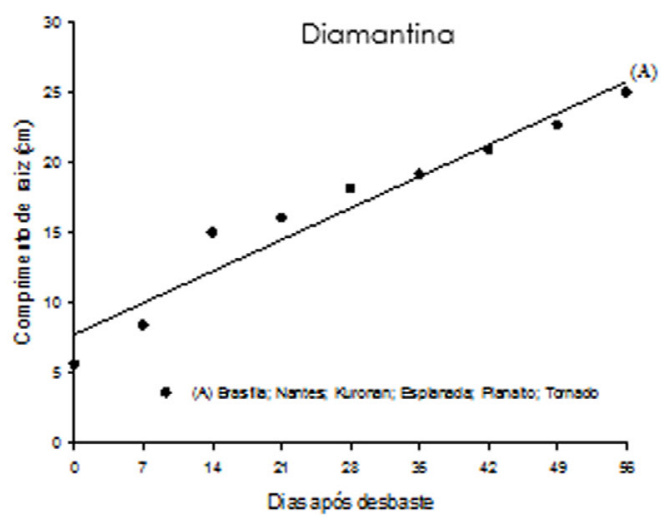

( $\mathrm{Vl})$

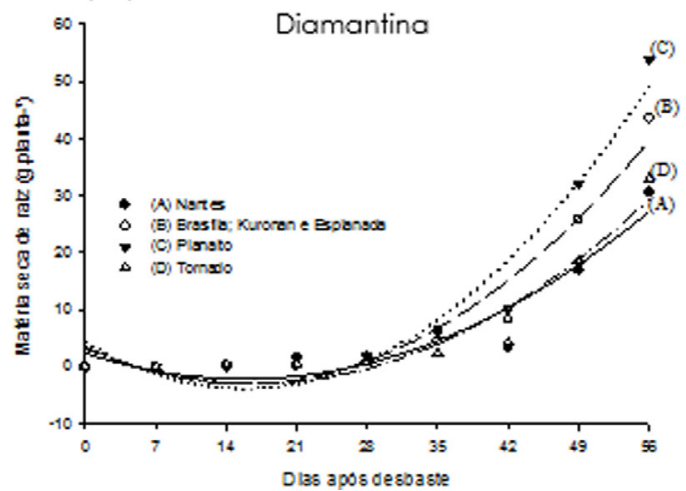

Figura 1. Altura da parte aérea (I e II), comprimento (III e IV) e matéria seca de raiz (V e VI), em Couto de Magalhães de Minas e Diamantina, ao longo do ciclo de crescimento dos cultivares de cenoura. UFV JM, 2014. 
Tabela 3. Equações de regressão das variáveis altura da parte aérea (ALT), comprimento de raiz (COMP), matéria seca de raiz (MSR), matéria seca total (MST), produtividade (PROD) e índice de colheita (IC) de diferentes cultivares de cenoura em Couto Magalhães de Minas e Diamantina. UFV JM, 2014.

\begin{tabular}{|c|c|c|c|c|}
\hline Local & Variável & Modelos ajustados & $\mathrm{R}^{2}$ & Cultivares* \\
\hline \multirow{13}{*}{$\begin{array}{c}\text { Couto de Magalhães } \\
\text { de Minas }\end{array}$} & \multirow{2}{*}{ ALT } & $\hat{Y}=7,304+0,921 x$ & 0,9706 & $1,3,4,5,6$ \\
\hline & & $\hat{Y}=4,236+0,855 x$ & 0,9883 & 2 \\
\hline & \multirow{3}{*}{ COMP } & $\hat{Y}=3,82634+0,503 x-0,0036 x^{2}$ & 0,9875 & $1,3,5$ \\
\hline & & $\hat{Y}=4,262+0,54692 x-0,0042 x^{2}$ & 0,9361 & 4 \\
\hline & & $\hat{Y}=2,476+0,559875 x-0,004 x^{2}$ & 0,9458 & 2,6 \\
\hline & \multirow{2}{*}{ MSR } & $\hat{Y}=2,215-0,74227 x+0,0286 x^{2}$ & 0,9775 & $1,4,5$ \\
\hline & & $\hat{Y}=1,5327-0,5403 x+0,0213 x^{2}$ & 0,9610 & $2,3,6$ \\
\hline & \multirow{2}{*}{ MST } & $\hat{Y}=1,8003-0,7386 x+0,0346 x^{2}$ & 0,9696 & $1,3,4,5$ \\
\hline & & $\hat{Y}=1,573-0,56343 x+0,0243 x^{2}$ & 0,9557 & 2,6 \\
\hline & \multirow{3}{*}{ PROD } & $\hat{Y}=0,889-0,3562 x+0,01891 x^{2}$ & 0,9975 & 1 \\
\hline & & $\hat{Y}=2,7196-0,6976 x+0,0246 x^{2}$ & 0,9683 & $2,4,6$ \\
\hline & & $\hat{Y}=2,88-0,794848 x+0,0307 x^{2}$ & 0,9814 & 3,5 \\
\hline & IC & $\hat{Y}=0,14016+1,35596 x$ & 0,9610 & Todas \\
\hline \multirow{15}{*}{ Diamantina } & \multirow{2}{*}{ ALT } & $\hat{Y}=5,988+1,0214 x-0,00913 x^{2}$ & 0,9843 & $1,3,4,5$ \\
\hline & & $\hat{Y}=4,514+0,9215 x-0,00787 x^{2}$ & 0,9831 & 2,6 \\
\hline & COMP & $\hat{Y}=7,753+0,321 x$ & 0,9371 & Todas \\
\hline & \multirow{4}{*}{ MSR } & $\hat{Y}=2,401-0,5738 x+0,01816 x^{2}$ & 0,9112 & 2 \\
\hline & & $\hat{Y}=3,5323-0,8281 x+0,0262 x^{2}$ & 0,9423 & $1,3,4$ \\
\hline & & $\hat{Y}=4,3227-1,0325 x+0,0327 x^{2}$ & 0,9448 & 5 \\
\hline & & $\hat{Y}=3,1043-0,7134 x+0,0211 x^{2}$ & 0,9229 & 6 \\
\hline & \multirow{4}{*}{ MST } & $\hat{Y}=3,822-0,8546 x+0,0302 x^{2}$ & 0,9526 & $1,3,4$ \\
\hline & & $\hat{Y}=2,3075-0,5266 x+0,019 x^{2}$ & 0,9130 & 2 \\
\hline & & $\hat{Y}=4,16997-0,974 x+0,0351 x^{2}$ & 0,9568 & 5 \\
\hline & & $\hat{Y}=2,9357-0,65665 x+0,022 x^{2}$ & 0,9515 & 6 \\
\hline & \multirow{3}{*}{ PRODT } & $\hat{Y}=1,180-0,3567 x+0,01791 x^{2}$ & 0,9916 & $1,3,4,6$ \\
\hline & & $\hat{Y}=0,6745-0,25475 x+0,013 x^{2}$ & 0,9637 & 2 \\
\hline & & $\hat{Y}=1,3149-0,4541 x+0,0228 x^{2}$ & 0,9824 & 5 \\
\hline & IC & $\hat{Y}=1,07063+1,43362 x$ & 0,9795 & Todas \\
\hline
\end{tabular}

*1 - Brasilia; 2 - Nantes; 3 - Kuronan; 4 - Esplanada; 5 - Planalto e 6 - Tornado 
(l)

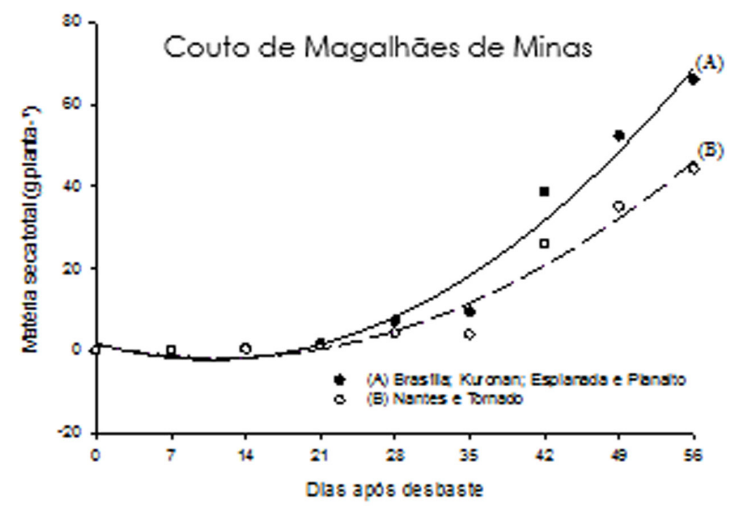

(III)

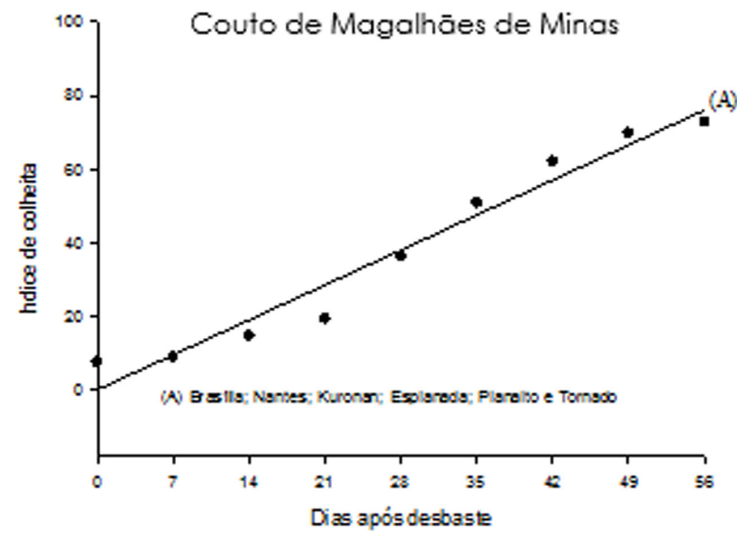

(V)

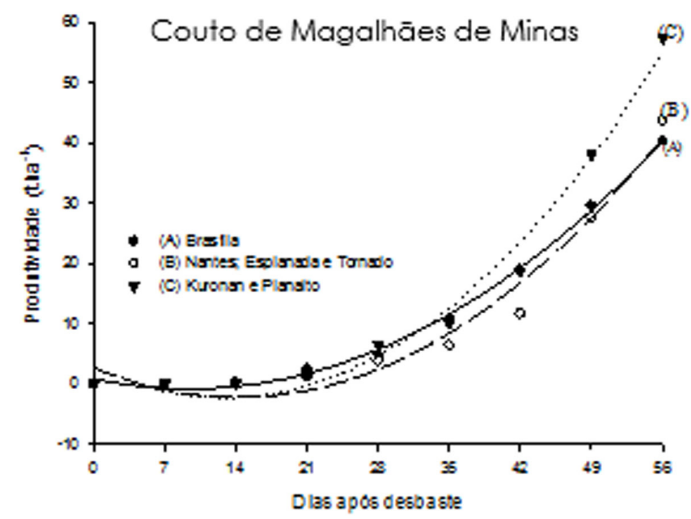

(II)

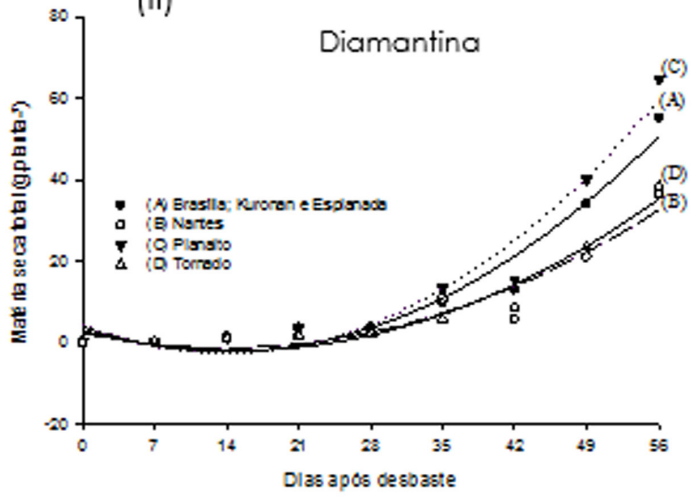

(IV)

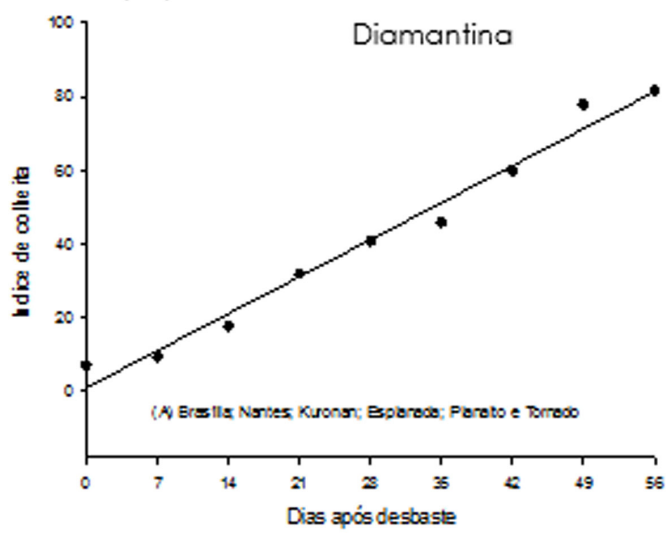

(VI)

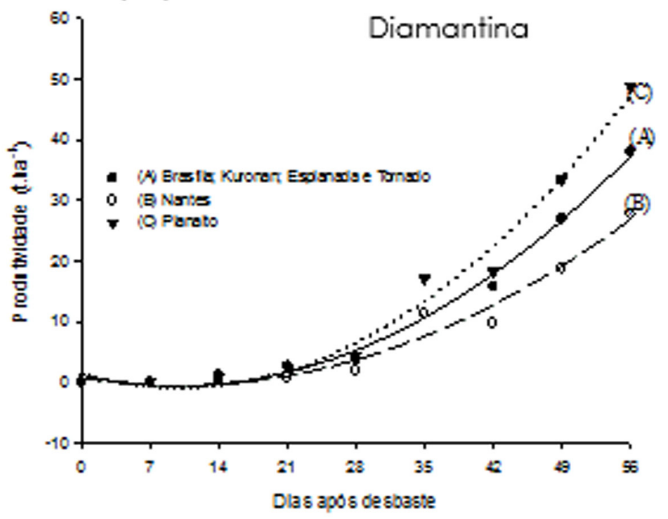

Figura 2. Matéria seca total (I e II), índice de colheita (III e IV) e produtividade (V e VI), em Couto de Magalhães de Minas e Diamantina, ao longo do ciclo de crescimento dos cultivares

Brasília, Esplanada e Planalto, apresentando maior discrepância nos resultados a partir dos 28 dias após desbaste (Figura $1 \mathrm{~V}$ e $1 \mathrm{VI}$ ). O mesmo comportamento foi observado em Diamantina para o cultivar Planalto e posteriormente Brasília, Kuronan e Esplanada, que se destacaram em relação a Nantes e Tornado a partir dos 35 DAD.

Em Couto de Magalhães de Minas, os cultivares Kuronan e Planalto apresentaram maiores produtividades, com cerca de $55 \mathrm{tha}^{-1}$.
Os demais genótipos foram semelhantes, cerca de $40 \mathrm{tha}^{-1}$. A diferença foi que a cv. Brasília, alcançou maiores acúmulos na fase inicial de cultivo, enquanto as demais, só se igualaram a ela no final do ciclo. Isso demonstra que esse cultivar permite uma colheita antecipada, caso haja alguma especificidade onde esta prática se torne interessante, como preços altos no mercado.

Em Diamantina, novamente o cultivar 
Planalto foi a mais produtiva (Figura $2 \mathrm{~V}$ e 2VI) (Tabela 3). Vieira et al. (2012) também encontraram altos valores de produção de raízes para a cv. Planalto nos ensaios realizados em Brasília-DF, evidenciando o grande potencial produtivo deste cultivar. Ainda em Diamantina, a cv. Nantes apresentou a menor produtividade entre todos os tratamentos estudados, sendo o único tratamento a apresentar produtividade inferior à média nacional, cerca de $27 \mathrm{t} \mathrm{ha}^{-1}$. Resultados semelhantes foram encontrados nos ensaios realizados por Luz et al. (2009) em Uberlândia-MG, onde também foi observado que este material foi o de menor desempenho produtivo.

\section{Conclusões}

Ambientes distintos resultam em diferentes comportamentos dos cultivares de cenoura, sendo que em Couto de Magalhães de Minas os cultivares apresentaram maiores índices produtivos.

O cultivar Planalto é mais indicada para plantio em Diamantina e a Kuronan e a Planalto para Couto Magalhães de Minas.

\section{Agradecimentos}

Ao Conselho Nacional de Desenvolvimento Científico e Tecnológico (CNPq), Fundação de Amparo à Pesquisa do Estado de Minas Gerais (FAPEMIG) e a Coordenação de Aperfeiçoamento de Pessoal de Nível Superior (CAPES) pelo financiamento e suporte para a execução do trabalho.

\section{Referências}

Andrade, A.C., Fonseca, D.M., Lopes, R.S., Nascimento Júnior, D., Cecon, P.R., Queiroz, D.S., Pereira, D.H., Reis, S.T. 2005. Análise de crescimento do capim-elefante 'napier' adubado e irrigado. Ciência e Agrotecnologia 29: 415-423.

Bezerra, A.M.E., Medeiros Filho, S., oliveira, L.D.M., Silveira, E.R. 2008. Produção e composição química da macela em função da época de colheita. Horticultura Brasileira 26: 327-332.

CEAGESP. Cenoura: no caminho da modernização: relatório do ano de 2000, São Paulo: Programa brasileiro para a melhoria dos padrões comerciais e embalagens de hortigranjeiros. 2000. http://www.hortibrasil. org.br/jnw/images/stories/folders/cenoura.pdf <Acesso em 21 de Fev. 2014>
CFSEMG. 1999. Recomendações para O uso de corretivos e fertilizantes em Minas Gerais: $5^{a}$ Aproximação. UFV, Viçosa, Brasil. 359 p.

Collares, G.L., Reinert, D.J., Reichert, J.M., Kaiser, D.R. 2008. Compactação de um Latossolo induzida pelo tráfego de máquinas e sua relação com o crescimento e produtividade de feijão e trigo. Revista Brasileira de Ciência do Solo 32: 933-942.

Cruz, C.D., Castoldi, F.L. 1991. Decomposição da interação genótipos $x$ ambientes em partes simples e complexa. Revista Ceres 38: 422-430.

Embrapa Hortaliças. Irrigação da Cultura da Cenoura, Brasília: Embrapa hortaliças. 2007. http://www.agencia.cnptia.embrapa.br/ Repositorio/ct_48_000gvxvkk9k02wx7ha0g934vgr wmjhcl.pdf<Acesso em 21 Ago. 2014>.

Embrapa Hortaliças. Hortaliças em números: relatório do ano de 2008, Brasília: Embrapa hortaliças. 2008. http://www.cnph.embrapa. br/paginas/hortalicas_em_numeros/balanca_ comercial_1997_2008.xis<Acesso em 21 Fev. $2014>$.

Figueiredo, E.B., Malheiros, E.B., Braz, L.T. 2004. Interação genótipo $\mathrm{x}$ ambiente em cultivares de alface na região de Jaboticabal. Horticultura Brasileira 22: 66-71.

Floss, E. 2006. Fisiologia de plantas cultivadas: o estudo que está por trás do que se vê. UPF, Passo Fundo, Brasil. $751 \mathrm{p}$.

Fontes, P.C.R., Dias, E.N., Silva, D.J.H. 2005. Dinâmica do crescimento, distribuição de matéria seca e produção de pimentão em ambiente protegido. Horticultura Brasileira 23: 9499.

Gualberto, R. 2002. Produtividade, adaptabilidade e estabilidade fenotípica de cultivares de tomateiro sob diferentes condições de ambiente. Pesquisa Agropecuária Brasileira 37: 81-88.

Lopes, W.A.R., Negreiros, M.Z., Teófilo, T.M.S., Alves, S.S.V., Martins, C.M., Nunes, G.H.S., Grangeiro, L.C. 2008. Produtividade de cultivares de cenoura sob diferentes densidades de plantio. Revista Ceres 55: 482-487.

Luz, J.M.Q., Silva Júnior, J.A., Teixeira, M.S.S.C., Silva, M.A.D., Severino, G.M., Melo, B. 2009. Desempenho de cultivares de cenoura no verão e outono-inverno em Uberlândia-MG. Horticultura Brasileira 27: 96-99.

Machado, E.C., Schmidt, P.T., Medina, C.L., Ribeiro, R.V. 2005. Respostas da fotossíntese de três espécies de citros a fatores ambientais. Pesquisa Agropecuária Brasileira 40: 1161-1170. 
Oliveira C.D., Braz, L.T., Banzatto, D.A. 2005. Adaptabilidade e estabilidade fenotípica de genótipos de cenoura. Horticultura Brasileira 23: 743-748.

Peixoto, N., Filgueira, F.A.R., Melo, P.E., Buso, J.A., Monteiro, J.D., Braz, L.T., Purquerio, L.F.V., Hamasaki, R.I. 2002. Seleção de clones de batata para microclimas de altitude no Planalto Central. Horticultura Brasileira 20: 438-441.

Possas, J.M.C., Correa, M.M., Moura, G.B.A., Lopes, P.M.O., Caldas, A.M., Fontes Júnior, R.V.P. 2012. Zoneamento agroclimático para a cultura do pinhão-manso no Estado de Pernambuco. Revista Brasileira de Engenharia Agrícola e Ambiental 16: 993-998.

Regazzi, A.J., Silva, C.H.O. 1999. Teste para verificar a identidade de modelos de regressão e a igualdade de parâmetros no caso de dados de delineamentos experimentais. Revista Ceres 46: 383-409.

Reinert, D.J., Albuquerque, J.A., Reichert, J.M., Aita, C., Andrada, M.M.C. 2008. Limites críticos de densidade do solo para o crescimento de raízes de plantas de cobertura em Argissolo Vermelho. Revista Brasileira de Ciência do Solo 32: 1805-1816.

Resende, F.V., Souza, L.S., Oliveira, P.S.R., Gualberto, R. 2005. Uso de cobertura morta vegetal no controle da umidade e temperatura do solo, na incidência de plantas invasoras e na produção da cenoura em cultivo de verão. Ciência e Agrotecnologia 29: 100-105.

Richart, A., Tavares Filho, J., Brito, O.R., Llanillo, R.F., Ferreira, R. 2005. Compactação do solo: causas e efeitos. Semina: Ciências Agrárias 26: 321-344.

Vieira, J.V., Silva, G.O., Boiteux, L.S. 2012. Genetic parameter and correlation estimates of processing traits in half-sib progenies of tropicaladapted carrot germplasm. Horticultura Brasileira 30: 7-11. 